\title{
Editorial zum Schwerpunkt: Interdisziplinäre Aspekte und Fragen an der Schwelle zum Tod
}

Insbesondere in der strafrechtlichen Praxis wird auf Verständigungsschwierigkeiten und Verständnisprobleme zwischen Juristen und medizinischen Sachverständigen hingewiesen. Als Gründe für die »Krise des medizinischen Sachverständigenbeweises« sowie für die »Zwangsehe« zwischen forensischen Sachverständigen auf der einen und Juristen auf der anderen Seite werden u. a. die unterschiedlichen Fachsprachen sowie andere Denk- und Arbeitsweisen angeführt. Sprachliche Missverständnisse auf der Fachebene gipfeln bisweilen in sprachlichen Missgriffen auf der persönlichen Ebene, um von fehlender Professionalität auch im Umgang mit dem eigenen fehlenden Wissen von der Fachsprache und Arbeitsweise des anderen abzulenken oder um ein unerwünschtes Ergebnis zu verhindern.

Zur Lösung des Konflikts wird eine verbesserte interdisziplinäre Weiterbildung sowie der obligatorische Erwerb von rechtsmedizinischem und forensisch-psychiatrischem Grundlagenwissen von Juristen auch schon während des Studiums gefordert. Eine enge Kooperation und ausführliche interdisziplinäre Kommunikation zwischen Juristen und Medizinern fördert deshalb die Bereitschaft, die andere Profession verstehen zu wollen und verstehen zu können.

An der Johann Wolfgang Goethe-Universität in Frankfurt wird seit einiger Zeit eine enge Zusammenarbeit zwischen der Humanmedizin und der Rechtswissenschaft durch die Durchführung gemeinsamer medizinrechtlicher Seminare gepflegt. Hierdurch sollen Studierenden der beiden Fachbereiche Denk- und Arbeitsweisen vermittelt werden, um im gemeinsamen Dialog Stärken und Schwächen der eigenen Fachdisziplin sowie die unterschiedlichen Herangehensweisen an rechtliche und medizinische Problemstellungen erkennen und gegebenenfalls lösen zu können.

Das Themenheft entstand im Rahmen dieser interdisziplinären Kooperation und widmet sich im Schwerpunkt medizinrechtlichen Fragestellungen am Lebensende aus rechtlicher, medizinischer, aber auch ethisch-philosophischer Sicht. Während Gesetzgeber und Exekutive in zahlreichen Rechtsgebieten eine wahre Regelungsflut entfalten, sind wichtige Fragen an der Schwelle zum Tod normativ ungeklärt.

Den in diesem Schwerpunktheft gebündelten Beiträgen ist das Anliegen gemeinsam, den Gesetzgeber zu behutsamer, aber deutlicher Aktion zu ermuntern, zumal die Regelungsbeispiele nur wenigen Spezialisten bekannt, dafür aber für jedermann relevant sind. Reinhard Dettmeyer und Burkhard Madea, Rechtsmediziner aus Bonn, zeichnen ein deutliches und zugleich erschreckendes Mängelprofil des Leichenschauund Obduktionsrechtes. Es fehlt in der Bundesrepublik Deutschland eine notwendige Vereinheitlichung dieses Rechtsbereichs, wodurch die Bestimmung von Todesursachen häufig einer »Black Box« ähnelt. Besonders beklagt werden fehlende Regelungen für therapeutische Gewebeentnahmen anlässlich von Obduktionen, wobei die ohnehin niedrige Obduktionsquote das Fehlen der ärztlichen Qualitätskontrolle zum Nutzen der Lebenden zu vereiteln droht.

Hansjürgen Bratzke, Markus Parzeller und Claudia Henze aus der Rechtsmedizin der Johann Wolfgang Goethe-Universität in Frankfurt thematisieren verfehlte und pra- 
xisferne Regelungen im Transplantationsgesetz, das erst 1997 in Kraft trat und erhebliche Schwachstellen aufweist: die unzureichende Differenzierung des Gesetzgebers bei Organ- und Gewebeentnahmen, die fehlende Praktikabilität des im Transplantationsgesetz festgeschriebenen Verfahrens zur Gewebeentnahme, die Frage der Todesfeststellung und die normativ unklare Regelung der Auskunftspflichten. Auch bei der Lebendspende fordern die Autoren eine gesetzgeberische Klarstellung. Die Kriminalisierung von Organspendern, Organempfängern und Ärzten habe erneut zur Abschreckung, Unsicherheit und Ablehnung gegenüber der Organspende und der Transplantationsmedizin geführt.

Markus Parzeller, Arzt und Jurist am Institut für Forensische Medizin der Universität Frankfurt, setzt sich mit einem normativen Defizit auseinander, das wesentliche Bereiche am Ende des Lebens in unerträglicher Unklarheit belässt. Es bedürfe einer gesetzgeberischen Konkretisierung der Todeszeitpunkte und einer diesbezüglichen Festlegung durch den demokratisch legitimierten Gesetzgeber, wann der Mensch als tot anzusehen ist.

Der Medizinethiker Arnd T. May aus Bochum analysiert die bislang vorliegenden Vorschläge für den Gesetzgeber zur Regelung von Patientenverfügungen. Sicherung und Schutz der Selbstbestimmung des Einzelnen sollten Gegenstände gesellschaftlicher Diskussion und gesetzgeberischen Bemühens sein. Insbesondere weist der Autor auf den Stellenwert von unterstützenden Angeboten bei der Information, Beratung und Erstellung von Patientenverfügungen hin. Erst dadurch würde »Gesundheitsmündigkeit« hergestellt, die Voraussetzung für Wahrnehmung von Autonomie sei. Der Richter Jürgen Seichter aus Nidda fächert im Schlussbeitrag diesbezügliche Sachverhalte auf, die die praktische Umsetzung von Wünschen auf Behandlungsabbruch mit lebensbeendender Zielsetzung betreffen. Der Rechtspraktiker gibt analysierend zu bedenken, dass Grenzsituationen, wie die Überprüfung, ob einem Wunsch auf Behandlungsabbruch entsprochen werden kann, sich weitgehend der für das rechtliche Denken erforderlichen Typisierung und Generalisierung entzögen. Aus diesem Grunde sei ein vorgegebenes förmliches Verfahren allein nicht sachgerecht und sollte weder dem Richter noch dem Beteiligten aufgenötigt werden - so der Praktiker. Besondere Bedeutung wird der Patientenverfügung zugesprochen, die aufgrund eines eingehenden Aufklärungsgesprächs mit einem behandelnden Arzt verfaßt und von diesem mit unterzeichnet wird. Bestimmungen in Patientenverfügungen sollten möglichst konkret und klar sein, absolute Bindungswirkung könne eine Patientenverfügung aber auch nicht beanspruchen. Die Möglichkeit einer Änderung der vormaligen Meinung könne nie ausgeschlossen werden.

Derartige Fragen und Probleme an der Schwelle zum Tod bedürfen behutsamer normativer Pflege und steten intensiven interdisziplinären Austausches, denn »ewige Wahrheiten « gibt es nicht. Kritische wissenschaftliche Beobachtung und Diskussion bleiben daher dauerhafte Aufgaben.

Frankfurt am Main im Dezember 2004

Peter-Alexis Albrecht

Hansjürgen Bratzke

Markus Parzeller 